\title{
Thermal lens measurements in the cornea
}

\author{
SHALINI VENKATESH,' SHEENA GUTHRIE,?'FRANCIS R CRUICKSHANK,? \\ RAYMOND T BAILEY, ' WALLACE S FOULDS, ' AND WILLIAM R LEE'
}

From the 'Tennent Institute of Ophthalmology, University of Glasgow; and the 'Department of Pure and Applied Chemistry, University of Strathclyde, Glasgow

SUMmARY Q-switched pulses from a neodymium/YAG (yttrium-aluminium-garnet) laser were passed through corneal discs taken from the enucleated eyes of three baboons and four rabbits. The time course of heat dissipation following absorption of laser energy by the tissue was studied with the use of a second continuous wave laser beam acting as a probe. It was found that the absorption of each neodymium/YAG pulse created a transient divergent lens within the cornea as theoretical considerations predicted. The relaxation time that characterised the decay of this thermal lens for a 1/e laser beam diameter of $2.0 \mathrm{~mm}$ was found to be $2 \cdot 3 \pm 0 \cdot 1 \mathrm{~s}$ (mean \pm standard error for 12 separate groups of measurements). Our results show that Q-switched laser pulses passing through apparently unaffected transparent tissues can induce thermal lens effects which persist for several seconds. The optical transfer of each pulse in a stream will be identical only if enough time is left between pulses for the tissues to return to their initial state. Therefore, when such laser pulses sharply focused to perform high precision intraocular surgery are used, thermal lensing in the transparent ocular media must limit the rate at which pulses can be usefully delivered.

Thermal lens effects were first discovered in 1964 by Gordon et al. in the course of studies on Raman scattering in liquids. They subsequently developed a model that could quantitatively explain their observations.' Our current understanding of the phenomenon may be simply summarised as follows.

Thermal lensing is the creation of variations in refractive index within a virtually transparent medium following the passage of a laser beam through it. It occurs because absorption of energy from a laser beam passing through a substance, which may be solid, liquid, or gas, raises the temperature locally in a pattern that matches the intensity profile across the width of the laser beam. This quickly leads to corresponding density changes and thus to changes in refractive index. For many materials the refractive index is lowered with temperature, and this reduction must be greatest at the centre of the laser beam where the light intensity is highest. In effect a diverging lens is formed within and by the absorbing substance. The focal length of the lens depends on the nature of the substance and on the laser pulse's power. With time, if no further laser radiation is passed through, all the

Correspondence to Dr S Venkatesh. Tennent Institute of Ophthalmology, University of Glasgow, Glasgow G11 6NT. absorbed energy is thermally dissipated into the bulk of the substance, and the thermal lens effect is lost. The rate of loss depends on the thermal properties of the substance and the diameter of the laser beam. Fig. 1 illustrates the formation of a thermal lens.

To date thermal lensing has been studied only in gases, liquids, and relatively simple non-biological solids. Now, however, thermal lens effects are of interest in relation to high precision eye surgery by lasers. Recently there has been a lot of interest in the use of short duration, high power laser pulses directed into the chamber angle in the treatment of open-angle glaucoma ${ }^{2}$ and in the disruption of vitreal bands, opacified lens capsules, etc. ${ }^{3}$ Any such application requires precise focusing, and, although the fraction of laser energy that would be absorbed by the cornea is likely to be small, significant thermal lensing has been found to occur within substances whose absorption coefficients are lower than $10^{-3} \mathrm{~cm}{ }^{i} \cdot{ }^{4}$ It was, therefore, theoretically possible that a laser pulse passing through corneal tissue would create a thermal lens, which would have the effect of defocusing subsequent laser pulses. This investigation was designed to discover whether such lensing really did occur, and, if it did, to follow the time course of its decay. 


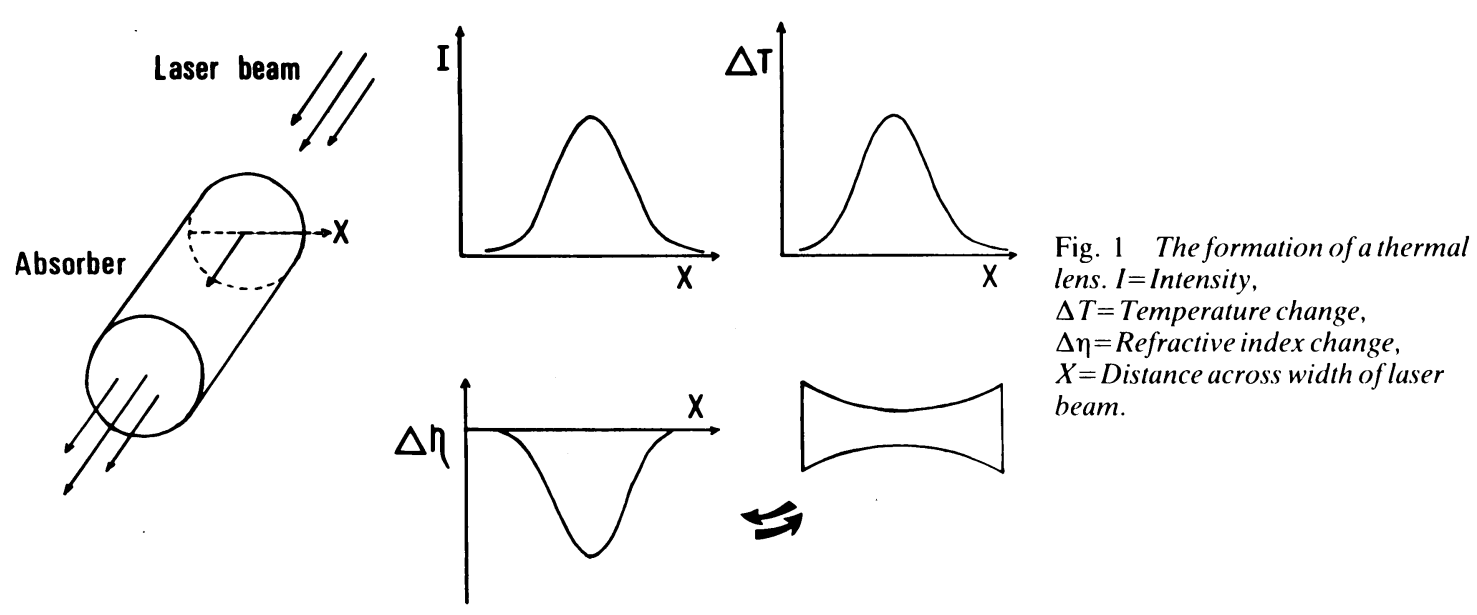

\section{Materials and methods}

Unfixed eyes were enucleated immediately after death from four rabbits and three baboons, animals which had been used in other quite separate experiments undertaken by coworkers. The corneal tissue from these animals is physically very similar to that of humans. The eyes were then immersed in chilled Barany's solution and transported to the laser laboratory within 30 minutes. Discs of corneal and limbal tissue were excised from these eyes. The scleral rim of each corneal disc was attached to a metal ring with Histoacryl Blue tissue adhesive and placed within a transparent cell filled with Barany's solution (Fig. 2). The experimental apparatus was arranged so that the parallel output beam of a pulsed neodymium/YAG laser (JK Lasers Ltd, Hyper-YAG 2000 system) would pass through the central thickness of the cornea and then be diverted into an absorbing block. To test for thermal lensing a second laser beam, from a low power continuous wave helium neon laser, was passed coaxially through the cornea. This tracer beam was then directed through a pinhole aperture and a narrow band pass filter on to a photomultiplier tube connected to a transient recorder (Biomation Corp., Biomation 1010) and microcomputer (Apple Computer Inc., Apple II).

With this arrangement if a thermal lens was formed in the cornea by a YAG pulse it would diverge the initially parallel, Gaussian profiled $\mathrm{HeNe}$ beam and reduce the intensity detected at the pinhole aperture of the photomultiplier. Initially the pinhole was positioned on the axis of the helium neon beam, where the steady intensity detected was a maximum. The possibility that an apparent thermal lens signal was in fact due to a lateral shift of the $\mathrm{HeNe}$ beam was excluded in the following way. As soon as such a signal was detected for the first time the pinhole aperture was moved to each of a set of slightly off axis positions in turn and a YAG pulse fired each time. It

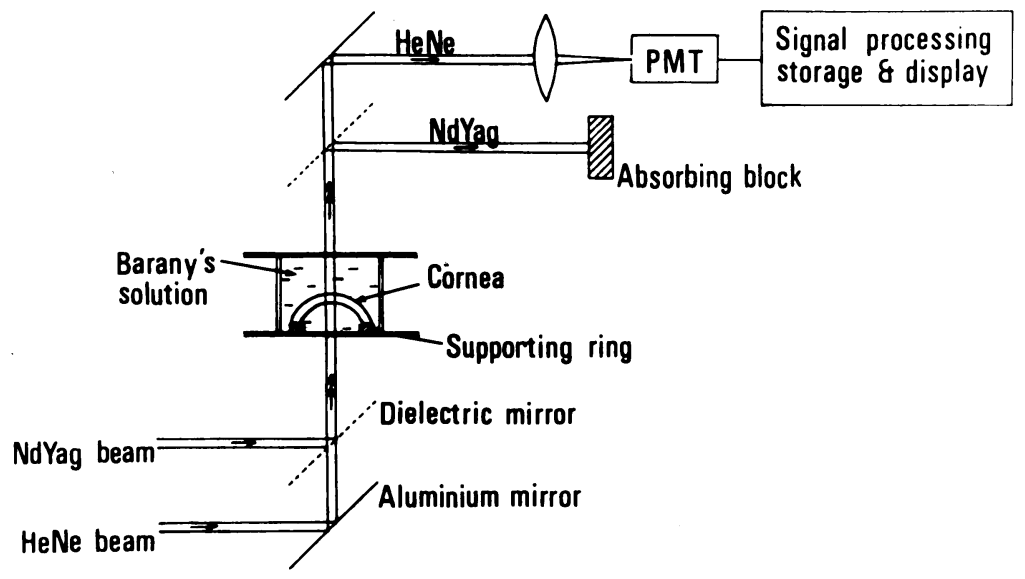

Fig. 2 Schematic diagram of experimental apparatus. 
was ascertained that the PMT signal was indeed smaller at each position around the original axial one. The first signal must, therefore, have been a true lensing signal signifying divergence of the beam rather than any lateral shift.

The YAG laser was set up to lase in its fundamental transverse mode, confirmed by direct measurements with a photodiode array (EG and G Reticon, RL $512 \mathrm{G}$ ), and at its fundamental wavelength of 1.06 $\mu \mathrm{m}$. Pulses were of energy 18 to $22 \mathrm{~mJ}$ and duration 25 to $30 \mathrm{~ns}$, giving power densities at the cornea of approximately $10^{5}$ to $10^{6} \mathrm{Watt} / \mathrm{mm}^{2}$ for a $1 / \mathrm{e}$ beam diameter of $2 \cdot 0 \mathrm{~mm}$.

It was ascertained by trial and error that a $10 \mathrm{~s}$ gap between the YAG pulses was long enough for the effect of a single YAG pulse to be observed independently of the effect of any other, that is, $10 \mathrm{~s}$ was long enough for recovery of the tissue from thermal lensing effects-vi. A stream of these pulses was thus fired at $10 \mathrm{~s}$ intervals (over a period of 15 to 20 minutes), and the signal from the photomultiplier was recorded after each pulse. As each individual signal was very small the signal to noise ratio of the measurement was improved by averaging automatically all the signals collected through this time. However, the final signal still represented the effect of a single, if 'typical', YAG pulse.

At least one set of 100 or so results was obtained from each cornea tested. In five instances a second set was obtained by slightly repositioning the cornea to use an adjacent area. Before trying any corneal measurements trial runs were carried out in the sample cell with water, chloroform, and Barany's solution in turn. The first two liquids were chosen as their thermal characteristics have been well established in the past. Barany's solution was tested, as it would be present during the corneal measurements.

\section{Results}

Fig. 3 shows a single, typical thermal lens signal after a single YAG pulse was passed through the test cell.

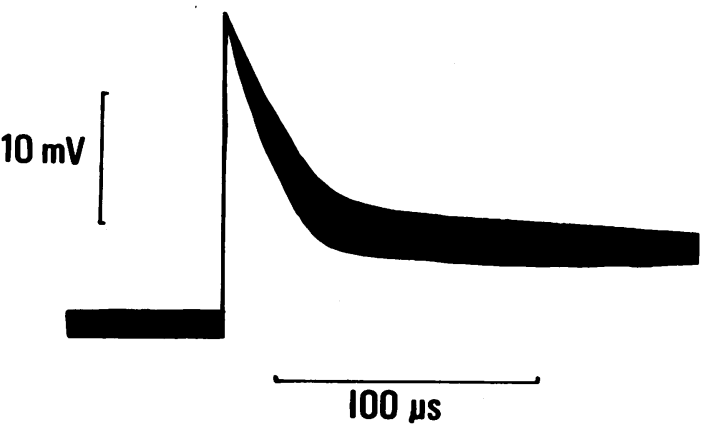

Fig. 3 A typical thermal lens trace from chloroform.
This particular trace, the inverse of the $\mathrm{HeNe}$ intensity versus time, was recorded from chloroform, but the same general pattern of a very sharp rise and a slow decline was found with the other liquids tried and with the corneae.

Our main interest in this study was the time dependent decay of the thermal lens signals. The signals were sampled, recorded, and analysed by the microcomputer. It used a program based on a model developed and tested by Bailey et al.in 1980 and $1981 . .^{56}$ They showed that the time dependence during the period of recovery, when heat conduction acts to dissipate the lensing effect, is determined by a relation time parameter. This parameter $\tau$ is characteristic of the absorbing substance concerned and varies with the square of the laser beam diameter. Their full mathematical analysis showed that the thermal lens signal $S$ of the tracer beam is related to $\tau$ by the following equation:

$$
1 / \sqrt{\mathrm{S}} \approx \mathrm{t} / \mathrm{\tau c}+1 / \mathrm{c} \ldots \ldots \ldots \ldots \ldots \ldots
$$

where $t$ is time and $\mathrm{c}$ is independent of time and the laser parameters. So if $t=5 \tau$ say, $S_{5 \tau} \approx c^{2} / 36 c$.f. at $t=0$, $\mathrm{S}_{0} \approx \mathrm{c}^{2}$

So, after a time of $5 \tau$, the thermal lens signal would drop to about $3 \%$ of its peak value.

A plot of the left hand side of equation 1 against time $t$ should, therefore, give a straight line whose intercept divided by slope will yield the value of $\tau$.

In applying this analysis to our study of lensing in corneal tissue this plot and calculation were carried out automatically for each signal within each set of 100 or so measurements. Some runs were carried out

Table 1 . Values of relaxation time $\tau$ obtained for corneae and some liquids

\begin{tabular}{|c|c|c|c|}
\hline Sample: & $\begin{array}{l}\text { Beam } \\
\text { diameter } \\
(\mathrm{mm})\end{array}$ & $\begin{array}{l}\text { Measured } \\
\tau(s)\end{array}$ & $\begin{array}{l}\tau \text { Corrected to } \\
\text { beam diameter } \\
\text { of } 2.0 \mathrm{~mm}\end{array}$ \\
\hline Water & 0.9 & 0.359 & $1 \cdot 77$ \\
\hline Water & $1 \cdot 5$ & $1 \cdot 00$ & $1 \cdot 77$ \\
\hline Water & $2 \cdot 0$ & $1 \cdot 80$ & $1 \cdot 80$ \\
\hline Barany's solution & 0.9 & $0 \cdot 369$ & $1 \cdot 82$ \\
\hline Chloroform & 0.9 & $0 \cdot 616$ & $3 \cdot(04$ \\
\hline \multicolumn{4}{|l|}{ Cornea } \\
\hline Baboon 1 & $1 \cdot 5$ & $1 \cdot 1$ & 1.9 \\
\hline Baboon 1 & $1 \cdot 5$ & $1 \cdot 6$ & $2 \cdot 8$ \\
\hline Rabbit 1 & $1 \cdot 5$ & $1 \cdot 4$ & $2 \cdot 5$ \\
\hline Rabbit 1 & $1 \cdot 5$ & $1 \cdot 7$ & $3 \cdot()$ \\
\hline Baboon 2 & $1 \cdot 5$ & $1 \cdot 2$ & $2 \cdot 1$ \\
\hline Baboon 3 & $2 \cdot()$ & 1.9 & 1.9 \\
\hline Baboon 3 & $2 \cdot 0$ & $1 \cdot 7$ & $1 \cdot 7$ \\
\hline Rabbit 2 & $2 \cdot 0$ & $2 \cdot 6$ & $2 \cdot 6$ \\
\hline Rabbit 3 & $2 \cdot 0$ & $2 \cdot 8$ & $2 \cdot 8$ \\
\hline Rabbit 3 & $2 \cdot()$ & $2 \cdot 7$ & $2 \cdot 7$ \\
\hline Rabbit 4 & $2 \cdot 0$ & $2 \cdot 0$ & $2 \cdot 0$ \\
\hline Rabbit 4 & $2 \cdot 0$ & $1 \cdot 5$ & $1 \cdot 5$ \\
\hline
\end{tabular}


at a YAG beam diameter of $1.6 \mathrm{~mm}$ and others at $2.00 \mathrm{~mm}$, but as the dependence of $\tau$ on beam diameter was known the values of $\tau$ were all corrected to a common beam diameter of $2.0 \mathrm{~mm}$. These corrected values of $\tau$ are given in Table 1 .

For water and Barany's solution values of $\tau$ varying from $1.77 \mathrm{~s}$ to $1.80 \mathrm{~s}$ were obtained. For corneae values lay between $1.5 \mathrm{~s}$ and $2.8 \mathrm{~s}$ with a mean of $2.3 \mathrm{~s}$ $\pm 0.1 \mathrm{~s}$ (standard error of the mean for 12 sets of measurements). For chloroform a value of $3.04 \mathrm{~s}$ was obtained.

\section{Discussion}

Thermal lensing has been shown to occur with the $1.06 \mu \mathrm{m}$ beam of a neodymium/YAG laser in water, Barany's solution, and chloroform, and values of the relaxation time parameter $\tau$ obtained in each case. When corneal tissue immersed in Barany's solution was tested signals were obtained which persisted for a time significantly longer than that from the solution alone and so must have resulted from lensing in the tissue itself. The experiments have, therefore, shown that corneae do exhibit thermal lensing, and that recovery from lensing due to a laser beam diameter of $2.0 \mathrm{~mm}$ is characterised by a relaxation time of $2 \cdot 3 \mathrm{~s}$.

The values of wavelength, pulse energy, and power density at the cornea chosen for this study are typical of those obtained with Q-switched neomymium/YAG lasers now used in experimental and clinical eye surgery. ${ }^{*}$ One application of such a laser which is of particular interest to us is laser trabeculotomy as a possible treatment of open-angle glaucoma. This is one example of the use of sharply focused Q-switched pulses in ocular surgery which could be adversely affected by the relatively long decay of thermal lensing in corneal tissue. For example, if our laser system were set up to fire one pulse per second the thermal lens created in the cornea by the first pulse will not have had time to decay before the second

\footnotetext{
${ }^{*}$ Consider the Sirius-Microruptor 2 system, for example, one of the first and best known Q-switched Nd/YAG laser systems produced commercially for ophthalmic applications. Data supplied by the manufacturer, LASAG AG of Switzerland, show an expected beam area at the cornea of $6 \mathrm{~mm}^{2}$, which for pulses of up to $70 \mathrm{~mJ}$ delivered in $12 \mathrm{~ns}$ means corneal power densities of up to $10^{6} \mathrm{watt} / \mathrm{mm}^{2}$. Compare the laser system used in this study which gave, typically, 20 $\mathrm{mJ}$ delivered in $35 \mathrm{~ns}$ over a corncal area of $3 \mathrm{~mm}^{2}$, corresponding to a corneal power density of $2 \cdot 10^{5} \mathrm{watt} / \mathrm{mm}^{2}$.
}

pulse reaches it. The second pulse will then be focused at a point slightly beyond that of the first pulse. The shift of focus will increase for the third pulse and so on. Only if a time of say $5 \tau$ or more is left between one pulse and the next will the defocusing effect be lost.

In fact, reducing the laser beam diameter at the cornea from the $2.0 \mathrm{~mm}$ we chose for our experiments would reduce the relaxation time and so allow pulses to be fired at at a higher rate without defocusing, but in practice a diameter of at least this size is necessary to avoid damaging the cornea at the same time as the target tissue.

The magnitude of the defocusing due to thermal lensing in the cornea cannot be predicted from the results of these particular experiments, but there is some outside evidence that it may be of practical significance. Fankhauser et al. stated in a recent paper on laser surgery ${ }^{7}$ that the focus of their YAG laser, pulsed at a frequency of $50 \mathrm{~Hz}$, shifted by up to $3 \mathrm{~mm}$ according to the number of pulses fired in one burst, and that an empirical correction had to be applied when aiming. The authors gave no explanation of this defocusing effect, but the results of our experiments, and in particular the large measured values of corneal relaxation time, suggest that such defocusing could well be due, at least in part, to thermal lensing within the cornea.

This study was supported by the Rank Prizc Fund (Grant No 7269).

\section{References}

1 Gordon JP, Leite RCC, Moore RS, Whinnery JR. Long-transient effects in lasers with inserted liquid samples. J Appl Physiol 1965; 36: 3 .

2 Van der Zypen E. Fankhauser F. Lasers in the treatment of chronic simplc glaucoma. Trans Ophthalmol Soc UK 1982; 102: 147.

3 Aron-Rosa D, Griesemann JC, Aron JJ. Applications ophtalmologiques des lasers neodymium YAG pulses. J Fr Ophtalmol 1981;4: 61 .

4 Solimini D. Loss measurements of organic materials at $6328 \AA$. J Appl Physiol 1966: 37: 3314

5 Bailcy RT, Cruickshank FR, Pugh D. Johnstonc W. Pulsed source thermal Iens Part 1-theoretical analysis. J Chem Soc (Faraday 2) 1980; 76: 633.

6 Bailcy RT, Cruickshank FR, Pugh D, Johnstone W. Pulsed source thermal lens. Part 2: Experimental tests of the theory. $J$ Chem Soc (Faraday 2) 1981; 77: 1387.

7 Fankhauser F, Roussel P, Steffen J, Van der Zypen E, Chrenkova A. Clinical studies on the efficiency of high power laser radiation upon some structures of the anterior segment of the eye. Int Ophthalmol 1981; 3: 129. 